Mathematical Modelling and AnAlysis

Volume 17 Number 3, June 2012, 309-329

http://dx.doi.org/10.3846/13926292.2012.685955

(c) Vilnius Gediminas Technical University, 2012
Publisher: Taylor\&Francis and VGTU

http://www.tandfonline.com/TMMA

Print ISSN: 1392-6292

Online ISSN: 1648-3510

\title{
Classical Solution for Initial-Boundary Value Problem for Wave Equation with Integral Boundary Condition
}

\author{
Victor Korzyuk, Victor Erofeenko and Julia Sheika \\ Belarusian State University \\ Nezavisimosti av. 4, 220030 Minsk, Belarus \\ E-mail: korzyuk@bsu.by; erofeenko@bsu.by \\ E-mail(corresp.): pulko@tut.by
}

Received September 8, 2010; revised March 20, 2012; published online June 1, 2012

\begin{abstract}
The unique existence of classical solution of initial-boundary value problem for wave equation with a special integral boundary condition is proved in the work. Classical solution of the problem in analytical form is also found in the article. This problem arises at the modeling of electromagnetic fields with arbitrary time dependence when interaction between the field and solids is simulated with impedance boundary conditions.
\end{abstract}

Keywords: initial-boundary value problem, integral boundary condition, characteristic method.

AMS Subject Classification: 35G16; 35L05.

\section{Introduction}

Method of boundary value problems for modelling processes in electrodynamics is strongly connected with formulation of boundary conditions on media interface. Boundary conditions describe interaction processes between field and interfaces and essentially depend on a field time mode and material structure of near-surface layer. Models with impedance boundary conditions on strongly conductive bodies are widely used in applied electrodynamics. Impedance boundary conditions allow us to exclude the field calculation inside bodies. Such an approach is used when material medium of conductive body has complex physical and geometrical structure. In this case exact field calculation inside the body is connected with solution of two-domain boundary-value problem with classical boundary conjugation conditions. A solution of the problem becomes simpler when the effect of body on resultant electromagnetic field is modelled by impedance boundary condition.

Shchukin-Leontovich impedance boundary conditions are used for modelling electromagnetic fields with harmonic time-dependance $[2,7,13]$. In case 
of arbitrary time-dependance impedance boundary conditions take an integral form $[2,7,18]$. Problems for hyperbolic equations with non-local integral condition are thoroughly examined lately. Articles $[3,4,5,8,14,15,16,17]$ are devoted to analysis of solvability of non-local problems for hyperbolic equations with integral boundary conditions of different types with respect to space variable.

The purpose of this work is to examine the existence and unique solvability of classical solution of initial-boundary value problem with integral with respect to time boundary condition of special kind and of certain physical meaning and to find this solution.

\section{Definition of Initial-Boundary Value Problem for Wave Equation with Integral with Respect to Time Boundary Condition}

Let $\mathbb{R}^{3}$ be a Euclidean space with fixed Cartesian coordinates $O x y z$ and basis $\vec{e}_{x}, \vec{e}_{y}, \vec{e}_{z}$. We put plane screen $D=\{0<x<l,-\infty<y, z<\infty\}$, filled with material with permittivity $\varepsilon$, magnetic permeability $\mu$ and conductivity $\gamma=0$ into $\mathbb{R}^{3}$ space (Fig. 1). In half-space $D_{1}(x<0)$ medium is characterized by $\varepsilon_{1}, \mu_{1}, \gamma_{1}$. There is electromagnetic field $\vec{E}=\vec{E}(x, y, z, t), \vec{H}=\vec{H}(x, y, z, t)$ penetrating in layer $D$.

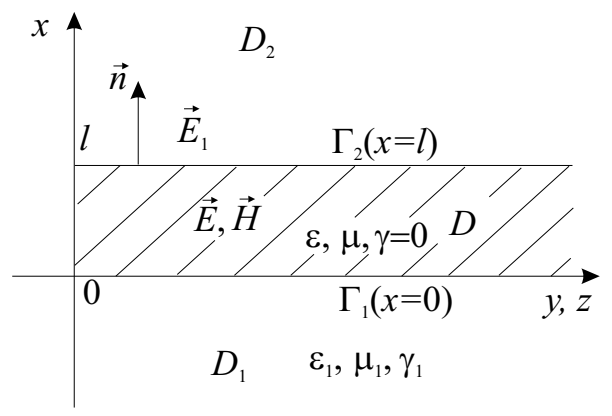

Figure 1. Plain screen.

Electromagnetic field satisfies Maxwell's equations:

$$
\operatorname{rot} \vec{H}=\varepsilon \frac{\partial \vec{E}}{\partial t}, \quad \operatorname{rot} \vec{E}=-\mu \frac{\partial \vec{H}}{\partial t}, \quad t>0,(x, y, z) \in D
$$

Electromagnetic field $\vec{E}, \vec{H}$ is generated by initial conditions

$$
\left.\vec{E}\right|_{t=0}=\vec{E}_{0},\left.\quad \vec{H}\right|_{t=0}=\vec{H}_{0}, \quad(x, y, z) \in D
$$

and given tangential component of electric field on plain $\Gamma_{2}(x=l)$

$$
\left.[\vec{n},[\vec{E}, \vec{n}]]\right|_{x=l}=\vec{E}_{1}, \quad t>0,-\infty<y, z<\infty,
$$


where $\vec{E}_{0}=\vec{E}_{0}(x, y, z), \vec{H}_{0}=\vec{H}_{0}(x, y, z)$ are electric and magnetic field intensity at initial time $t=0 ; \vec{E}_{1}=\vec{E}_{1}(x, y, t)$ is vector tangent to plain $\Gamma_{2}$.

We assume that field and medium of half-space $D_{2}(x>l)$ do not interact and penetration of electromagnetic field from layer $D$ into half-space $D_{1}$ is modeled by impedance boundary condition that does not take into account displacement currents induced in $D_{1}[2]$ :

$$
\begin{aligned}
& {\left.[\vec{n}, \vec{E}]\right|_{x=0}=-\left.\sqrt{\frac{\mu_{1}}{\pi \gamma_{1}}} \int_{0}^{t} \frac{\partial}{\partial \eta}[\vec{n},[\vec{H}(x, y, z, \eta), \vec{n}]]\right|_{x=0} \frac{d \eta}{\sqrt{t-\eta}}, \quad t>0} \\
& -\infty<y, z<\infty
\end{aligned}
$$

where $\vec{n}=\vec{e}_{x}$ is normal to layer $D$.

It is necessary to find fields $\vec{E}, \vec{H}$ that satisfy equations (2.1)-(2.4). Electrodynamic problems with boundary condition (2.4) were studied in [5, 18].

Let electromagnetic field not depend on space coordinates $y, z$ and vectors $\vec{E}, \vec{H}, \vec{E}_{0}, \vec{H}_{0}, \vec{E}_{1}$ be parallel to layer $D$ of the kind:

$$
\vec{E}=E(x, t) \vec{e}_{y}, \quad \vec{H}=H(x, t) \vec{e}_{z}, \quad \vec{E}_{1}=\chi(t) \vec{e}_{y}, \quad \vec{E}_{0}=\varphi(x) \vec{e}_{y}, \quad \vec{H}_{0}=v_{0}(x) \vec{e}_{z} .
$$

Taking into consideration this assumption on electromagnetic field structure we transform Maxwell's equations (2.1) to wave equation. Hence we obtain the following scalar initial-boundary value problem with respect to unknown function $E(x, t)$ :

$$
\begin{aligned}
& \frac{\partial^{2} E(x, t)}{\partial t^{2}}-\frac{1}{\varepsilon \mu} \frac{\partial^{2} E(x, t)}{\partial x^{2}}=0, \quad t>0,0<x<l, \\
& \left.E(x, t)\right|_{t=0}=\varphi(x),\left.\quad \frac{\partial E(x, t)}{\partial t}\right|_{t=0}=-\frac{1}{\varepsilon} \frac{\partial v_{0}(x)}{\partial x}, \quad 0 \leqslant x \leqslant l, \\
& \left.E(x, t)\right|_{x=0}=\left.\frac{1}{\mu} \sqrt{\frac{\mu_{1}}{\pi \gamma_{1}}} \int_{0}^{t} \frac{\partial E(x, \tau)}{\partial x}\right|_{x=0} \frac{d \tau}{\sqrt{t-\tau}}, \quad t \geqslant 0, \\
& \left.E(x, t)\right|_{x=l}=E_{1}(t), \quad t \geqslant 0 .
\end{aligned}
$$

Let us find solution of the problem (2.5). We shall change some variables and formulate the problem in the following form.

Problem 1. Find function $u(x, t) \in C^{2}(\bar{\Omega}), \Omega=\{0<t<\infty, 0<x<l\}$ that satisfies conditions:

$$
\begin{aligned}
& \frac{\partial^{2} u(x, t)}{\partial t^{2}}-a^{2} \frac{\partial^{2} u(x, t)}{\partial x^{2}}=0 \quad \text { in } \Omega, \\
& \left.u(x, t)\right|_{t=0}=\varphi(x),\left.\quad \frac{\partial u(x, t)}{\partial t}\right|_{t=0}=\psi(x), \quad 0 \leqslant x \leqslant l, \\
& \left.u(x, t)\right|_{x=0}=\left.c \int_{0}^{t} \frac{\partial u(x, \tau)}{\partial x}\right|_{x=0} \frac{d \tau}{\sqrt{t-\tau}}+g(t), \\
& \left.u(x, t)\right|_{x=l}=\chi(t), \quad t \geqslant 0,
\end{aligned}
$$

where $\varphi(x) \in C^{2}([0, l]), \psi(x) \in C^{1}([0, l]), \chi(t) \in C^{2}([0, \infty)), g(t) \in C^{2}([0, \infty))$, $a=$ const, $a>0, c=$ const, $c>0$. 
Note that problems for hyperbolic equations with non-local boundary conditions are often considered in literature recently. Particularly works $[3,4,5,8$, $12,15,16,17]$ study solvability of non-local problems for hyperbolic equations with different integral conditions with respect to space coordinate.

\section{Solution of Auxiliary Problem for Initial-Boundary Va- lue Problem for Wave Equation with Integral Boundary Condition in Orthogonal Domain}

Let us consider auxiliary initial-boundary value problem in domain $\Omega_{b}=$ $\{b<t<b+l / a, 0<x<l\}$ (see, $[6,9,10,11])$.

Problem 2. It is necessary to find function $u^{(b)} \in C^{2}\left(\bar{\Omega}_{b}\right)$ that satisfies conditions:

$$
\begin{aligned}
& \frac{\partial^{2} u^{(b)}(x, t)}{\partial t^{2}}-a^{2} \frac{\partial^{2} u^{(b)}(x, t)}{\partial x^{2}}=0 \quad \text { in } \Omega_{b} \\
& \left.u^{(b)}(x, t)\right|_{t=b}=\varphi^{(b)}(x),\left.\quad \frac{\partial u^{(b)}(x, t)}{\partial t}\right|_{t=b}=\psi^{(b)}(x), \quad 0 \leqslant x \leqslant l \\
& \left.u^{(b)}(x, t)\right|_{x=0}=\left.c \int_{b}^{t} \frac{\partial u^{(b)}(x, \tau)}{\partial x}\right|_{x=0} \frac{d \tau}{\sqrt{t-\tau}}+g^{(b)}(t), \quad b \leqslant t \leqslant b+\frac{l}{a}, \\
& \left.u^{(b)}(x, t)\right|_{x=l}=\chi^{(b)}(t), \quad b \leqslant t \leqslant b+l / a
\end{aligned}
$$

where $\varphi^{(b)}(x) \in C^{2}([0, l]), \psi^{(b)}(x) \in C^{1}([0, l]), \chi^{(b)}(t) \in C^{2}\left(\left[b, b+\frac{l}{a}\right]\right), g^{(b)}(t) \in$ $C^{2}\left(\left[b, b+\frac{l}{a}\right]\right), a=$ const, $a>0, c=$ const, $c>0$.

Theorem 1. Let classical solution $u^{(b)}(x, t) \in C^{2}\left(\bar{\Omega}_{b}\right)$ of problem (3.1)-(3.4) exist and satisfy conditions

$$
\left.\frac{\partial^{3} u^{(b)}(x, t)}{\partial t^{2} \partial x}\right|_{x=0} \in \mathrm{L}_{1}\left(\left[b, b+\delta_{1}\right)\right), \quad \delta_{1}>0 .
$$

Let finite limit for the solution exist and have the form

$$
\left.\lim _{t \rightarrow b} \int_{b}^{t} \frac{\partial^{3} u^{(b)}(x, \tau)}{\partial x \partial \tau^{2}}\right|_{x=0} \frac{d \tau}{\sqrt{t-\tau}}=A,
$$

then coincidence conditions are fulfilled for initial and boundary functions in the angular point $(x, t)=(0, b)$ of domain $\bar{\Omega}_{b}$

$$
\varphi^{(b)}(0)=g^{(b)}(b), \quad \psi^{(b)}(0)=g^{(b) \prime}(b), \quad \varphi^{(b) \prime}(0)=0, \quad \psi^{(b) \prime}(0)=0,
$$

and in the angular point $(x, t)=(l, b)$ the following conditions are fulfilled

$$
\varphi^{(b)}(l)=\chi^{(b)}(b), \quad \psi^{(b)}(l)=\chi^{(b) \prime}(b), \quad \chi^{(b) \prime \prime}(b)=a^{2} \varphi^{(b) \prime \prime}(l) .
$$


Proof. Let us proof fulfilment of coincidence conditions at the point $(0, b)$. We consider the first initial condition (3.2) when $x=0$ and boundary condition (3.3) when $t \rightarrow b$

$$
\varphi^{(b)}(0)=\left.\lim _{t \rightarrow b} u^{(b)}(x, t)\right|_{x=0}=\left.c \lim _{t \rightarrow b} \int_{b}^{t} \frac{\partial u^{(b)}(x, \tau)}{\partial x}\right|_{x=0} \frac{d \tau}{\sqrt{t-\tau}}+g^{(b)}(b) .
$$

As $u^{(b)}(x, t)$ is the solution of the problem (3.1)-(3.4) hence $\frac{\partial u^{(b)}(x, t)}{\partial x} \in C\left(\bar{\Omega}_{b}\right)$. Thus we obtain that $\left.\frac{\partial u^{(b)}(x, t)}{\partial x}\right|_{x=0}$ is bounded when $b \leqslant t \leqslant b+\delta_{1}$. It means that there is such a constant $M_{1}<\infty$ that $\left|\frac{\partial u^{(b)}(x, t)}{\partial x}\right|_{x=0} \mid \leqslant M_{1}$ and then

$$
\begin{aligned}
\left.\lim _{t \rightarrow b} \int_{b}^{t} \frac{\partial u^{(b)}(x, \tau)}{\partial x}\right|_{x=0} \frac{d \tau}{\sqrt{t-\tau}} & \leqslant \lim _{t \rightarrow b} \int_{b}^{t}\left|\frac{\partial u^{(b)}(x, \tau)}{\partial x}\right|_{x=0} \mid \frac{d \tau}{\sqrt{t-\tau}} \\
& \leqslant M_{1} \lim _{t \rightarrow b} \int_{b}^{t} \frac{d \tau}{\sqrt{t-\tau}}=0 .
\end{aligned}
$$

Hence we obtain $\varphi^{(b)}(0)=g^{(b)}(b)$.

Let us consider the second initial condition (3.2) when $x=0$ and the first boundary condition when $t \rightarrow b$

$$
\psi^{(b)}(0)=\left.\lim _{t \rightarrow b} \frac{\partial u^{(b)}(x, t)}{\partial t}\right|_{x=0}=c \lim _{t \rightarrow b} \frac{\partial G(t)}{\partial t}+\left.\frac{g^{(b)}(t)}{\partial t}\right|_{t=b},
$$

where

$$
G(t)=\left.\int_{b}^{t} \frac{\partial u^{(b)}(x, \tau)}{\partial x}\right|_{x=0} \frac{d \tau}{\sqrt{t-\tau}}
$$

We integrate function $G(t)$ by parts taking into account that $\frac{\partial u^{(b)}(x, b)}{\partial x}=$ $\varphi^{(b) \prime}(x)$, and thus we obtain

$$
G(t)=2 \sqrt{t-b} \varphi^{(b) \prime}(0)+\left.2 \int_{b}^{t} \frac{\partial^{2} u^{(b)}(x, \tau)}{\partial x \partial \tau}\right|_{x=0} \sqrt{t-\tau} d \tau
$$

We differentiate derived expression

$$
G^{\prime}(t)=\frac{\varphi^{(b) \prime}(0)}{\sqrt{t-b}}+\left.\int_{b}^{t} \frac{\partial^{2} u^{(b)}(x, \tau)}{\partial x \partial \tau}\right|_{x=0} \frac{d \tau}{\sqrt{t-\tau}}
$$

then coincidence condition (3.9) takes the form

$$
\psi^{(b)}(0)=\left.\frac{\partial g^{(b)}}{\partial t}\right|_{t=b}+c \lim _{t \rightarrow b}\left(\frac{\varphi^{(b) \prime}(0)}{\sqrt{t-b}}\right)+c \lim _{t \rightarrow b}\left(\left.\int_{b}^{t} \frac{\partial^{2} u^{(b)}(x, \tau)}{\partial x \partial \tau}\right|_{x=0} \frac{d \tau}{\sqrt{t-\tau}}\right) .
$$

As $u^{(b)}(x, t)$ is the solution of the problem (3.1)-(3.4) then $\frac{\partial^{2} u^{(b)}(x, t)}{\partial x \partial t} \in$ $C\left(\bar{\Omega}_{b}\right)$. Hence we obtain that $\left.\frac{\partial^{2} u^{(b)}(x, t)}{\partial x \partial t}\right|_{x=0}$ is limited when $b \leqslant t \leqslant b+\delta_{2}$, it 
means that there is such a constant $M_{2}<\infty$ that $\left|\frac{\partial^{2} u^{(b)}(x, t)}{\partial x \partial t}\right|_{x=0} \mid \leqslant M_{2}$, then

$$
\begin{aligned}
\lim _{t \rightarrow b}\left(\left.\int_{b}^{t} \frac{\partial^{2} u^{(b)}(x, \tau)}{\partial x \partial \tau}\right|_{x=0} \frac{d \tau}{\sqrt{t-\tau}}\right) & \leqslant \lim _{t \rightarrow b}\left(\int_{b}^{t}\left|\frac{\partial^{2} u^{(b)}(x, \tau)}{\partial x \partial \tau}\right|_{x=0} \mid \frac{d \tau}{\sqrt{t-\tau}}\right) \\
& \leqslant M_{2} \lim _{t \rightarrow b} \int_{b}^{t} \frac{d \tau}{\sqrt{t-\tau}}=0 .
\end{aligned}
$$

As $g^{(b)}(t)$ is generally an arbitrary function hence for time derivative $\frac{\partial u^{(b)}(x, t)}{\partial t}$ be determined at the point $(0, b)$ we suppose that

$$
\varphi^{(b) \prime}(0)=0,
$$

then coincidence condition (3.9) takes the form $\psi^{(b)}(0)=g^{(b) \prime}(b)$. Besides coincidence condition of the second kind should be fulfilled. Let us consider equation $(3.1)$ at the point $(0, b)$. Taking into account the first initial condition (3.2), the first boundary condition (3.3) and expression (3.10) we obtain

$$
c G^{\prime \prime}(b)+g^{(b) \prime \prime}(b)-a^{2} \varphi^{(b) \prime \prime}(0)=0 .
$$

Subject to condition (3.12) expression (3.11) takes the form

$$
G^{\prime}(t)=\left.\int_{b}^{t} \frac{\partial^{2} u^{(b)}(x, \tau)}{\partial x \partial \tau}\right|_{x=0} \frac{d \tau}{\sqrt{t-\tau}} .
$$

Let us transform function $G^{\prime}(t)$ integrating it by parts and taking into account that $\frac{\partial^{2} u^{(b)}(0, b)}{\partial x \partial t}=\psi^{(b) \prime}(0)$ :

$$
G^{\prime}(t)=2 \sqrt{t-b} \psi^{(b) \prime}(0)+\left.2 \int_{b}^{t} \frac{\partial^{3} u^{(b)}}{\partial x \partial \tau^{2}}\right|_{x=0} \sqrt{t-\tau} d \tau .
$$

We differentiate function $G^{\prime}(t)$ :

$$
G^{\prime \prime}(t)=\frac{\psi^{(b) \prime}(0)}{\sqrt{t-b}}+\left.\int_{b}^{t} \frac{\partial^{3} u^{(b)}(x, \tau)}{\partial x \partial \tau^{2}}\right|_{x=0} \frac{d \tau}{\sqrt{t-\tau}} .
$$

Let us find

$$
G^{\prime \prime}(b)=\lim _{t \rightarrow b}\left(\frac{\psi^{(b) \prime}(0)}{\sqrt{t-b}}\right)+\lim _{t \rightarrow b}\left(\left.\int_{b}^{t} \frac{\partial^{3} u^{(b)}(x, \tau)}{\partial x \partial \tau^{2}}\right|_{x=0} \frac{d \tau}{\sqrt{t-\tau}}\right) .
$$

As $\psi^{(b)}(x)$ is generally an arbitrary function hence for coincidence condition (3.13) be determined at the point $(0, b)$ when $c \neq 0$ we suppose that $\psi^{(b) \prime}(0)=$ 0. Taking into account conditions (3.6) we obtain $G^{\prime \prime}(b)=A$, and hence condition (3.13) takes the form

$$
a^{2} \varphi^{(b) \prime \prime}(0)=g^{(b) \prime \prime}(b)+c A .
$$

Let us prove coincidence condition at the point $(l, b)$. We consider the first initial condition (3.2) when $x=l$ and the second boundary condition (3.4) when $t=b$

$$
\varphi^{(b)}(l)=\left.\lim _{t \rightarrow b} u^{(b)}(x, t)\right|_{x=l}=\chi^{(b)}(b) .
$$


Considering the second initial condition (3.2) and the second boundary condition (3.4) we obtain

$$
\psi^{(b)}(l)=\left.\lim _{t \rightarrow b} \frac{\partial u^{(b)}(x, t)}{\partial t}\right|_{x=l}=\chi^{(b) \prime}(b) .
$$

Second-order coincidence condition should be fulfilled at the point $(l, b)$. Let us consider equation $(3.1)$ at the point $(l, b)$. Taking into account the first initial condition (3.2) and the second boundary condition (3.4) we obtain

$$
a^{2} \varphi^{(b) \prime \prime}(l)=\chi^{(b) \prime \prime}(b)
$$

Thus coincidence conditions (3.7), (3.8) are proved.

Let us find the solution of initial-boundary value problem (3.1)-(3.4). We consider characteristics of equation (3.1) in domain $\Omega_{b}$

$$
x-a t+a b=0, \quad l-x-a t+a b=0 .
$$

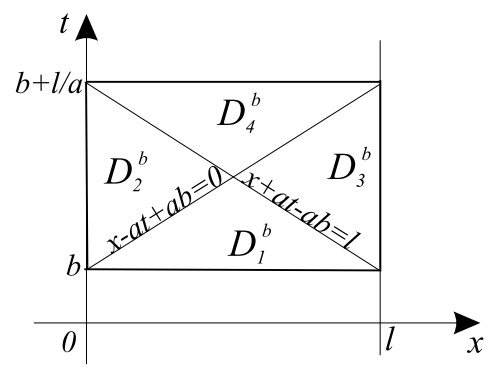

Figure 2. Division of domain $\Omega_{b}$ into four subdomains.

Characteristics divide domain $\Omega_{b}$ into four subdomains (see Fig. 2):

$$
\begin{aligned}
& D_{1}^{b}:\{(x, t) \mid b<t<b+l / 2 a, a t-a b<x<l+a b-a t\}, \\
& D_{2}^{b}:\{(x, t) \mid 0<x<l / 2, b+x / a<t<b+(l-x) / a\}, \\
& D_{3}^{b}:\{(x, t) \mid l / 2<x<l, b+(l-x) / a<t<b+x / a\}, \\
& D_{4}^{b}:\{(x, t) \mid b+l / 2 a<t<b+l / a, l+a b-a t<x<a t-a b\} .
\end{aligned}
$$

In each subdomain we solve equation (3.1) under its own boundary conditions.

Lemma 1. We suppose that $\varphi^{(b)}(x) \in C^{2}([0, l]), \psi^{(b)}(x) \in C^{1}([0, l]), \chi^{(b)}(t) \in$ $C^{2}([b, b+l / a]), g^{(b)}(t) \in C^{2}([b, b+l / a])$ and coincidence conditions

$$
\begin{aligned}
& \varphi^{(b)}(0)=g^{(b)}(b), \\
& \varphi^{(b)}(l)=\chi^{(b)}(b),
\end{aligned}
$$


are fulfilled. Then solution $u^{(b)}(x, t) \in C\left(\bar{\Omega}_{b}\right), u^{(b)}(x, t) \in C^{2}\left(D_{i}^{b}\right), i=\overline{1,4}$ of initial-boundary value problem (3.1)-(3.4) exists in domain $\Omega_{b}$ and takes the form

$$
u^{(b)}(x, t)=u_{j}^{(b)}(x, t) \quad \text { in } D_{j}^{b}, \quad j=1, \ldots, 4,
$$

where

$$
\begin{aligned}
& u_{1}^{(b)}(x, t)=\frac{\varphi^{(b)}(x-a t+a b)+\varphi^{(b)}(x+a t-a b)}{2}+\frac{1}{2 a} \int_{x-a t+a b}^{x+a t-a b} \psi^{(b)}(\xi) d \xi, \\
& u_{2}^{(b)}(x, t)=\frac{\varphi^{(b)}(x+a t-a b)-\varphi^{(b)}(a t-x-a b)}{2}+\frac{1}{2 a} \int_{a t-x-a b}^{x+a t-a b} \psi^{(b)}(\xi) d \xi \\
& +F^{(b)}(x-a t+a b), \\
& u_{3}^{(b)}(x, t)=\chi^{(b)}\left(\frac{x-l}{a}+t\right)+\frac{\varphi^{(b)}(x-a t+a b)-\varphi^{(b)}(2 l-x-a t+a b)}{2} \\
& +\frac{1}{2 a} \int_{x-a t+a b}^{2 l-x-a t+a b} \psi^{(b)}(\xi) d \xi \\
& u_{4}^{(b)}(x, t)=\chi^{(b)}\left(\frac{x-l}{a}+t\right)-\frac{\varphi^{(b)}(a t-x-a b)+\varphi^{(b)}(2 l-x-a t+a b)}{2} \\
& +\frac{1}{2 a} \int_{a t-x-a b}^{2 l-x-a t+a b} \psi^{(b)}(\xi) d \xi+F^{(b)}(x-a t+a b), \\
& F^{(b)}(y)=\frac{2 \sqrt{a}}{\pi c} g^{(b)}(b) \sqrt{-y}-\frac{2}{\pi c \sqrt{a}} \int_{0}^{y} g^{(b) \prime}\left(b-\frac{\xi}{a}\right) \sqrt{\xi-y} d \xi \\
& +\int_{0}^{y} e^{-\frac{a}{\pi c^{2}}(y-\xi)}\left\{\frac{a}{\pi c^{2}} g^{(b)}\left(b-\frac{\xi}{a}\right)-\varphi^{(b) \prime}(-\xi)-\frac{\psi^{(b)}(-\xi)}{a}\right\} \\
& \times \operatorname{erfc}\left(\frac{\sqrt{a(\xi-y)}}{\sqrt{\pi} c}\right) d \xi+g^{(b)}(b) e^{-\frac{a}{\pi c^{2}} y} \operatorname{erfc}\left(\frac{\sqrt{-a y}}{\sqrt{\pi} c}\right),
\end{aligned}
$$

$\operatorname{erfc}(x)=1-\operatorname{erf}(x), \operatorname{erf}(x)=\frac{2}{\sqrt{\pi}} \int_{0}^{x} e^{-\eta^{2}} d \eta-i s$ a probability integral [1]

Proof. General solution of equation (3.1) in domain $\Omega_{b}$ has the form

$$
u^{(b)}(x, t)=f_{1}(x-a t+a b)+f_{2}(l+a b-x-a t),
$$

where $f_{1}(x), f_{2}(x)$ are functions to determine.

Let us solve problem (3.1), (3.2) in domain $D_{1}^{b}$. General solution of equation (3.1) has the form (3.18). Let us satisfy initial conditions (3.2):

$$
\begin{aligned}
\left.u^{(b)}\right|_{t=b} & =f_{1}(x)+f_{2}(l-x)=\varphi^{(b)}(x), \\
\left.\frac{\partial u^{(b)}}{\partial t}\right|_{t=b} & =-a f_{1}^{\prime}(x)-a f_{2}^{\prime}(l-x)=\psi^{(b)}(x) .
\end{aligned}
$$


Integrating the second equation and solving obtained system we have

$$
\begin{aligned}
& f_{1}(x)=\frac{\varphi^{(b)}(x)}{2}-\frac{1}{2 a} \int_{0}^{x} \psi^{(b)}(\xi) d \xi-\frac{C}{2} \\
& f_{2}(x)=\frac{\varphi^{(b)}(l-x)}{2}+\frac{1}{2 a} \int_{0}^{l-x} \psi^{(b)}(\xi) d \xi+\frac{C}{2}
\end{aligned}
$$

where $C$ is an arbitrary constant. Substituting obtained expressions into general solution (3.18) we get solution of the problem (3.1), (3.2) in domain $D_{1}^{b}$ of the kind

$$
u_{1}^{(b)}(x, t)=\frac{\varphi^{(b)}(x-a t+a b)+\varphi^{(b)}(x+a t-a b)}{2}+\frac{1}{2 a} \int_{x-a t+a b}^{x+a t-a b} \psi^{(b)}(\xi) d \xi
$$

In domain $D_{2}^{b}$ we solve problem (3.1) under integral boundary condition (3.3) and condition on characteristic:

$$
\begin{aligned}
\left.u^{(b)}\right|_{x=a t-a b}= & u_{1}^{(b)}(a t-a b, t)=\frac{\varphi^{(b)}(0)+\varphi^{(b)}(2 a t-2 a b)}{2} \\
& +\frac{1}{2 a} \int_{0}^{2 a t-2 a b} \psi^{(b)}(\xi) d \xi, \quad b<t<b+\frac{l}{2 a} .
\end{aligned}
$$

General solution of equation (3.1) has the form (3.18). Let us satisfy boundary condition (3.3)

$$
\begin{aligned}
\left.u^{(b)}\right|_{x=0} & =f_{1}(a b-a t)+f_{2}(l+a b-a t) \\
& =g^{(b)}(t)+c \int_{b}^{t}\left\{f_{1}^{\prime}(a b-a \tau)-f_{2}^{\prime}(l+a b-a \tau)\right\} \frac{d \tau}{\sqrt{t-\tau}} .
\end{aligned}
$$

We make two changes of variables in obtained expression: $y=a b-a t$ and change in subintegral expression $\eta=a b-a \tau$ then expression takes the form

$$
f_{1}(y)+f_{2}(l+y)=g^{(b)}\left(b-\frac{y}{a}\right)-\frac{c}{\sqrt{a}} \int_{0}^{y}\left\{f_{1}^{\prime}(\eta)-f_{2}^{\prime}(l+\eta)\right\} \frac{d \eta}{\sqrt{\eta-y}} .
$$

Let us satisfy condition on characteristic (3.20). Taking into account coincidence condition (3.15) we obtain

$$
\begin{aligned}
\left.u^{(b)}\right|_{x=a t-a b} & =f_{1}(0)+f_{2}(l+2 a b-2 a t) \\
& =\frac{g^{(b)}(b)+\varphi^{(b)}(2 a t-2 a b)}{2}+\frac{1}{2 a} \int_{0}^{2 a t-2 a b} \psi^{(b)}(\xi) d \xi .
\end{aligned}
$$

We change variable $y=2 a b-2 a t$ in obtained expression then

$$
f_{2}(l+y)=\frac{g^{(b)}(b)+\varphi^{(b)}(-y)}{2}+\frac{1}{2 a} \int_{0}^{-y} \psi^{(b)}(\xi) d \xi-f_{1}(0) .
$$


Substituting expression (3.22) into (3.21) we obtain

$$
f_{1}(y)+\frac{c}{\sqrt{a}} \int_{0}^{y} f_{1}^{\prime}(\eta) \frac{d \eta}{\sqrt{\eta-y}}=G_{1}(y)
$$

where

$$
\begin{aligned}
G_{1}(y)= & f_{1}(0)-\frac{g^{(b)}(b)}{2}+g^{(b)}\left(b-\frac{y}{a}\right)-\frac{\varphi^{(b)}(-y)}{2}-\frac{1}{2 a} \int_{0}^{-y} \psi^{(b)}(\xi) d \xi \\
& -\frac{c}{2 \sqrt{a}} \int_{0}^{y}\left\{\varphi^{(b) \prime}(-\xi)+\frac{\psi^{(b)}(-\xi)}{a}\right\} \frac{d \xi}{\sqrt{\xi-y}} .
\end{aligned}
$$

Let us integrate expression (3.23) by $y$ in the range from 0 to $z$

$$
\int_{0}^{z} f_{1}(y) d y+\frac{c}{\sqrt{a}} \int_{0}^{z} \int_{0}^{y} f_{1}^{\prime}(\eta) \frac{d \eta}{\sqrt{\eta-y}} d y=\int_{0}^{z} G_{1}(y) d y .
$$

Changing order of integration in double integral and finding inner integral we get

$$
\int_{0}^{z} f_{1}(y) d y-\frac{2 c}{\sqrt{a}} \int_{0}^{z} f_{1}^{\prime}(\eta) \sqrt{\eta-z} d \eta=\int_{0}^{z} G_{1}(y) d y .
$$

We integrate the second integral by parts. Hence obtained expression takes the form

$$
\int_{0}^{z} f_{1}(y) d y+\frac{c}{\sqrt{a}} \int_{0}^{z} f_{1}(\eta) \frac{d \eta}{\sqrt{\eta-z}}=G_{2}(z)
$$

where

$$
\begin{aligned}
G_{2}(z)= & -\frac{2 c}{\sqrt{a}} f_{1}(0) \sqrt{-z}+\left(f_{1}(0)-\frac{g^{(b)}(b)}{2}\right) z \\
& -\frac{z}{2}\left(\varphi^{(b)}(-z)+\frac{1}{a} \int_{0}^{-z} \psi^{(b)}(\xi) d \xi\right)+\int_{0}^{z} g^{(b)}\left(b-\frac{\xi}{a}\right) d \xi \\
& +\frac{1}{2} \int_{0}^{z}\left\{\varphi^{(b) \prime}(-\xi)+\frac{\psi^{(b)}(-\xi)}{a}\right\}\left(-\xi+\frac{2 c}{\sqrt{a}} \sqrt{\xi-z}\right) d \xi
\end{aligned}
$$

We multiply expression (3.23) by $\frac{1}{\sqrt{y-z}}$ and integrate by $y$ from 0 to $z$

$$
\int_{0}^{z} f_{1}(y) \frac{d y}{\sqrt{y-z}}+\frac{c}{\sqrt{a}} \int_{0}^{z} \int_{0}^{y} f_{1}^{\prime}(\eta) \frac{d \eta}{\sqrt{\eta-y}} \frac{d y}{\sqrt{y-z}}=\int_{0}^{z} G_{1}(y) \frac{d y}{\sqrt{y-z}} .
$$

Changing order of integration in double integral and finding inner integral we get

$$
\int_{0}^{z} f_{1}(y) \frac{d y}{\sqrt{y-z}}=\frac{\pi c}{\sqrt{a}} f_{1}(z)+G_{3}(z)
$$

where

$$
\begin{aligned}
& G_{3}(z)=\frac{\pi c}{\sqrt{a}}\left(\frac{g^{(b)}(b)}{2}-f_{1}(0)\right)+2\left(g^{(b)}(b)-f_{1}(0)\right) \sqrt{-z} \\
& -\frac{\pi c}{2 \sqrt{a}}\left(\varphi^{(b)}(-z)+\frac{1}{a} \int_{0}^{-z} \psi^{(b)}(\xi) d \xi\right) \\
& -\int_{0}^{z}\left(\varphi^{(b) \prime}(-\xi)+\frac{\psi^{(b)}(-\xi)}{a}\right) \sqrt{\xi-z} d \xi+\int_{0}^{z} g^{(b)}\left(b-\frac{\xi}{a}\right) \frac{d \xi}{\sqrt{\xi-z}} .
\end{aligned}
$$


Substituting expression (3.25) into (3.24) we obtain

$$
f_{1}(z)+\frac{a}{\pi c^{2}} \int_{0}^{z} f_{1}(\xi) d \xi=G_{4}(z)
$$

where

$$
\begin{aligned}
& G_{4}(z)=\left(f_{1}(0)-\frac{g^{(b)}(b)}{2}\right)\left(1+\frac{a}{\pi c^{2}} z\right)-\frac{2 \sqrt{a}}{\pi c} g^{(b)}(b) \sqrt{-z} \\
& +\frac{a}{\pi c^{2}} \times \int_{0}^{z} g^{(b)}\left(b-\frac{\xi}{a}\right)\left(1-\frac{c}{\sqrt{a}} \frac{1}{\sqrt{\xi-z}}\right) d \xi \\
& +\frac{1}{2}\left(\varphi^{(b)}(-z)+\frac{1}{a} \int_{0}^{-z} \psi^{(b)}(\xi) d \xi\right) \times\left(1-\frac{a}{\pi c^{2}} z\right) \\
& +\frac{a}{2 \pi c^{2}} \int_{0}^{z}\left\{\varphi^{(b) \prime}(-\xi)+\frac{\psi^{(b)}(-\xi)}{a}\right\}\left(-\xi+\frac{4 c}{\sqrt{a}} \sqrt{\xi-z}\right) d \xi
\end{aligned}
$$

Equation (3.26) is a Volterra integral equation of the second kind. Let us solve it. We suppose that $f_{1}(z)=-\frac{a}{\pi c^{2}} C(z)+G_{4}(z)$ where $C(z)=\int_{0}^{z} f_{1}(\xi) d \xi$ and $C^{\prime}(z)=f_{1}(z), C(0)=0$. Thus solution of integral equation (3.26) is equivalent to solution of Cauchy problem for ordinary differential equation:

$$
C^{\prime}(z)+\frac{a}{\pi c^{2}} C(z)=G_{4}(z), \quad C(0)=0 .
$$

Solution of Cauchy problem has the form

$$
C(z)=\int_{0}^{z} e^{-\frac{a}{\pi c^{2}}(z-\xi)} G_{4}(\xi) d \xi
$$

then solution of integral equation (3.26) takes the form

$$
f_{1}(z)=-\frac{a}{\pi c^{2}} \int_{0}^{z} e^{-\frac{a}{\pi c^{2}}(z-\xi)} G_{4}(\xi) d \xi+G_{4}(z) .
$$

Taking into account expression $G_{4}(z)$ and performing some transformations we obtain

$$
\begin{aligned}
f_{1}(z)= & f_{1}(0)-\frac{g^{(b)}(b)}{2}+g^{(b)}(b) e^{-\frac{a}{\pi c^{2}} z} \operatorname{erfc}\left(\frac{\sqrt{-a z}}{\sqrt{\pi} c}\right) \\
& -\frac{\sqrt{a}}{\pi c} \int_{0}^{z} g^{(b)}\left(b-\frac{\xi}{a}\right) \frac{d \xi}{\sqrt{\xi-z}}-\frac{\varphi^{(b)}(-z)}{2}-\frac{1}{2 a} \int_{0}^{-z} \psi^{(b)}(\xi) d \xi \\
& +\int_{0}^{z} e^{-\frac{a}{\pi c^{2}}(z-\xi)}\left\{\frac{a}{\pi c^{2}} g^{(b)}\left(b-\frac{\xi}{a}\right)-\varphi^{(b) \prime}(-\xi)-\frac{\psi^{(b)}(-\xi)}{a}\right\} \\
& \times \operatorname{erfc}\left(\frac{\sqrt{a(\xi-z)}}{\sqrt{\pi} c}\right) d \xi .
\end{aligned}
$$


Calculating integral $\int_{0}^{z} g^{(b)}\left(b-\frac{\xi}{a}\right) \frac{d \xi}{\sqrt{\xi-z}}$ by parts and marking

$$
\begin{aligned}
F^{(b)}(y)= & \frac{2 \sqrt{a}}{\pi c} g^{(b)}(b) \sqrt{-y}-\frac{2}{\pi c \sqrt{a}} \int_{0}^{y} g^{(b) \prime}\left(b-\frac{\xi}{a}\right) \sqrt{\xi-y} d \xi \\
& +\int_{0}^{y} e^{-\frac{a}{\pi c^{2}}(y-\xi)}\left\{\frac{a}{\pi c^{2}} g^{(b)}\left(b-\frac{\xi}{a}\right)-\varphi^{(b) \prime}(-\xi)-\frac{\psi^{(b)}(-\xi)}{a}\right\} \\
& \times \operatorname{erfc}\left(\frac{\sqrt{a(\xi-y)}}{\sqrt{\pi} c}\right) d \xi+g^{(b)}(b) e^{-\frac{a}{\pi c^{2}} y} \operatorname{erfc}\left(\frac{\sqrt{-a y}}{\sqrt{\pi} c}\right)
\end{aligned}
$$

we obtain

$$
f_{1}(z)=f_{1}(0)-\frac{\varphi^{(b)}(-z)}{2}-\frac{1}{2 a} \int_{0}^{-z} \psi^{(b)}(\xi) d \xi+F^{(b)}(z) .
$$

Performing change of variable $z=l+y$ in expression (3.22) we get

$$
f_{2}(z)=\frac{g^{(b)}(b)-\varphi^{(b)}(l-z)}{2}+\frac{1}{2 a} \int_{0}^{l-z} \psi^{(b)}(\xi) d \xi-f_{1}(0) .
$$

Substituting expressions (3.27), (3.28) into solution (3.18) and performing transformations we derive solution of problem with integral boundary condition in domain $D_{2}^{b}$

$$
\begin{aligned}
u_{2}^{(b)}(x, t)= & \frac{\varphi^{(b)}(x+a t-a b)-\varphi^{(b)}(a t-x-a b)}{2} \\
& +\frac{1}{2 a} \int_{a t-x-a b}^{x+a t-a b} \psi^{(b)}(\xi) d \xi+F^{(b)}(x-a t+a b) .
\end{aligned}
$$

In domain $D_{3}^{b}$ we have to solve problem (3.1), (3.4) with condition on characteristic

$$
\begin{aligned}
\left.u^{(b)}\right|_{x=l+a b-a t}= & u_{1}^{(b)}(l+a b-a t, t)=\frac{\varphi^{(b)}(l-2 a t+2 a b)+\varphi^{(b)}(l)}{2} \\
& +\frac{1}{2 a} \int_{l-2 a t+2 a b}^{l} \psi^{(b)}(\xi) d \xi, \quad b<t<b+\frac{l}{2 a} .
\end{aligned}
$$

General solution of equation (3.21) has the form (3.18). Let us satisfy boundary condition (3.4)

$$
\left.u^{(b)}\right|_{x=l}=f_{1}(l-a t+a b)+f_{2}(a b-a t)=\chi^{(b)}(t) .
$$

Taking into account coincidence conditions (3.16) in condition on characteristic (3.30) we obtain

$$
\begin{aligned}
f_{1}(l+2 a b-2 a t)+f_{2}(0)= & \frac{\varphi^{(b)}(l+2 a b-2 a t)+\chi^{(b)}(b)}{2} \\
& +\frac{1}{2 a} \int_{l-2 a t+2 a b}^{l} \psi^{(b)}(\xi) d \xi .
\end{aligned}
$$


Classical Solution for Initial-Boundary Value Problem for Wave Equation321

Performing change of variable $y=l+2 a b-2 a t$ in expression (3.32) we get

$$
f_{1}(y)=\frac{\chi^{(b)}(b)}{2}-f_{2}(0)+\frac{\varphi(y)}{2}+\frac{1}{2 a} \int_{y}^{l} \psi(\xi) d \xi
$$

Taking into account (3.33) in (3.31) and performing change of variable we obtain

$$
f_{2}(y)=\chi^{(b)}\left(b-\frac{y}{a}\right)-\frac{\chi^{(b)}(b)}{2}+f_{2}(0)-\frac{\varphi^{(b)}(l+y)}{2}-\frac{1}{2 a} \int_{l+y}^{l} \psi^{(b)}(\xi) d \xi
$$

Substituting expressions (3.33), (3.34) into general solution (3.18) we derive solution in domain $D_{3}^{b}$

$$
\begin{aligned}
u_{3}^{(b)}(x, t)= & \chi^{(b)}\left(\frac{x-l}{a}+t\right)+\frac{\varphi^{(b)}(x-a t+a b)-\varphi^{(b)}(2 l-x-a t+a b)}{2} \\
& +\frac{1}{2 a} \int_{x-a t+a b}^{2 l-x-a t+a b} \psi^{(b)}(\xi) d \xi .
\end{aligned}
$$

In domain $D_{4}^{b}$ we solve problem (3.1) with conditions on characteristics

$$
\begin{aligned}
\left.u^{(b)}\right|_{x=a t-a b}= & u_{3}^{(b)}(a t-a b, t)=\chi^{(b)}(2 t-b-l / a) \\
& +\frac{\varphi^{(b)}(0)-\varphi^{(b)}(2 l-2 a t+2 a b)}{2}+\frac{1}{2 a} \int_{0}^{2 l-2 a t+2 a b} \psi^{(b)}(\xi) d \xi \\
\left.u^{(b)}\right|_{x=l-a t+a b}= & u_{2}^{(b)}(l-a t+a b, t)=\frac{\varphi^{(b)}(l)-\varphi^{(b)}(2 a t-l-2 a b)}{2} \\
& +\frac{1}{2 a} \int_{2 a t-l-2 a b}^{l} \psi^{(b)}(\xi) d \xi+F^{(b)}(l-2 a t+2 a b)
\end{aligned}
$$

General solution of equation (3.1) has the form (3.18). Let us satisfy condition on characteristic (3.36). Taking into account coincidence condition (3.15) we obtain

$$
\begin{aligned}
f_{1}(0)+f_{2}(l+2 a b-2 a t)= & \chi^{(b)}\left(2 t-b-\frac{l}{a}\right)+\frac{g^{(b)}(b)-\varphi^{(b)}(2 l-2 a t+2 a b)}{2} \\
& +\frac{1}{2 a} \int_{0}^{2 l-2 a t+2 a b} \psi^{(b)}(\xi) d \xi .
\end{aligned}
$$


Let us satisfy condition on characteristic (3.37). Taking into account coincidence condition (3.16) we obtain

$$
\begin{aligned}
f_{1}(l-2 a t+2 a b)+ & f_{2}(0)=\frac{\chi^{(b)}(b)-\varphi^{(b)}(2 a t-l-2 a b)}{2} \\
& +\frac{1}{2 a} \int_{2 a t-l-2 a b}^{l} \psi^{(b)}(\xi) d \xi+F^{(b)}(l-2 a t+2 a b) .
\end{aligned}
$$

By changing variable $y=l+2 a b-2 a t$ in expression (3.38) we obtain

$$
f_{2}(y)=\chi^{(b)}\left(b-\frac{y}{a}\right)-f_{1}(0)+\frac{g^{(b)}(b)-\varphi^{(b)}(l+y)}{2}+\frac{1}{2 a} \int_{0}^{l+y} \psi^{(b)}(\xi) d \xi .
$$

By changing variable $y=l+2 a b-2 a t$ in expression (3.39) we get

$$
f_{1}(y)=\frac{\chi^{(b)}(b)}{2}-f_{2}(0)-\frac{\varphi^{(b)}(-y)}{2}+\frac{1}{2 a} \int_{-y}^{l} \psi^{(b)}(\xi) d \xi+F^{(b)}(y) .
$$

Taking into account coincidence conditions (3.16) in (3.40) we obtain

$$
f_{2}(0)=\frac{g^{(b)}(b)+\chi^{(b)}(b)}{2}-f_{1}(0)+\frac{1}{2 a} \int_{0}^{l} \psi^{(b)}(\xi) d \xi
$$

Substituting expressions (3.40)-(3.42) into general solution (3.18) and performing transformations we derive solution in domain $D_{4}^{b}$

$$
\begin{aligned}
u_{4}^{(b)}(x, t)= & \chi^{(b)}\left(\frac{x-l}{a}+t\right)-\frac{\varphi^{(b)}(2 l-x-a t+a b)}{2}-\frac{\varphi^{(b)}(a t-x-a b)}{2} \\
& +\frac{1}{2 a} \int_{a t-x-a b}^{2 l-x-a t+a b} \psi^{(b)}(\xi) d \xi+F^{(b)}(x-a t+a b) .
\end{aligned}
$$

Thus we have found solution of initial-boundary value problem (3.1)-(3.4) in the form (3.19), (3.29), (3.35), (3.43). Solution is obviously twice continuously differentiable in domains $D_{i}^{b}, i=\overline{1,4}$. It is also obviously continuous on characteristics $x-a t+a b=0, l-x-a t+a b=0$. Hence $u^{(b)}(x, t) \in C\left(\bar{\Omega}_{b}\right)$, $u_{i}^{(b)}(x, t) \in C^{2}\left(D_{i}^{b}\right)$.

Let us show that solution continuity up to second-order derivatives remains on characteristics (3.14) when coincidence conditions (3.7), (3.8) are fulfilled.

Lemma 2. If $\varphi^{(b)}(x) \in C^{2}([0, l]), \psi^{(b)}(x) \in C^{1}([0, l]), \chi^{(b)}(t) \in C^{2}\left(\left[b, b+\frac{l}{a}\right]\right)$, $g^{(b)}(t) \in C^{2}\left(\left[b, b+\frac{l}{a}\right]\right)$ and there exists the solution $u^{(b)}(x, t)$ of initial-boundary value problem (3.1)-(3.4) of the kind (3.17) then fulfillment of coincidence conditions (3.7), (3.8) is sufficient for $u^{(b)}(x, t) \in C^{2}\left(\bar{\Omega}_{b}\right)$. 
Proof. Under conditions of lemma the conditions of Lemma 1 are satisfied. Thus $u^{(b)}(x, t) \in C\left(\bar{\Omega}_{b}\right)$ and $u_{i}^{(b)}(x, t) \in C^{2}\left(D_{i}^{b}\right), i=\overline{1,4}$. Let us show that $u^{(b)}(x, t) \in C^{2}\left(\bar{\Omega}_{b}\right)$. Comparing solutions $u_{1}(x, t)$ and $u_{2}(x, t)$ and their first and second-order derivatives between domains $D_{1}^{b}, D_{2}^{b}$ and also solutions $u_{3}(x, t)$ and $u_{4}(x, t)$ and their first and second-order derivatives between domains $D_{3}^{b}, D_{4}^{b}$ when $x=a t-a b$, we arrive at the conclusion that solution of initial-boundary value problem (3.1)-(3.4) is continuous on characteristic $x-a t-a b=0$ up to second-order derivatives.

Similarly comparing solutions $u_{1}(x, t)$ and $u_{3}(x, t)$ and their first and second-order derivatives between domains $D_{1}^{b}, D_{3}^{b}$ and also solutions $u_{2}(x, t)$ and $u_{4}(x, t)$ and their first and second-order derivatives between domains $D_{2}^{b}, D_{4}^{b}$ when $x=a t-a b+l$ we can draw a conclusion that solution of initial-boundary value problem (3.1)-(3.4) is continuous on characteristic $l-x-a t+a b=0$ up to second-order derivatives.

Hence solution of initial-boundary value problem (3.1)-(3.4) is continuous up to second-order derivatives in the whole domain $\Omega_{b}$ i.e. $u^{(b)}(x, t) \in C^{2}\left(\bar{\Omega}_{b}\right)$.

Lemma 3. If $\varphi^{(b)}(x) \in C^{2}([0, l]), \psi^{(b)}(x) \in C^{1}([0, l]), \chi^{(b)}(t) \in C^{2}\left(\left[b, b+\frac{l}{a}\right]\right)$, $g^{(b)}(t) \in C^{2}\left(\left[b, b+\frac{l}{a}\right]\right)$ and there exists the solution $u^{(b)}(x, t)$ of initial-boundary value problem (3.1)-(3.4) of the kind (3.17) then fulfilment of coincidence conditions (3.7), (3.8) is necessary for $u^{(b)}(x, t) \in C^{2}\left(\bar{\Omega}_{b}\right)$.

Proof. It is evident that $u^{(b)}(x, t) \in C^{2}\left(D_{i}^{b}\right), i=\overline{1,4}$. Let us find conditions necessary for solution $u^{(b)}(x, t)$ continuity up to second-order derivatives in the whole domain $\Omega_{b}$. We notice that $u^{(b)}(x, t)$ satisfies equation (3.1). Hence we obtain that conditions necessary for continuity of derivatives $\frac{\partial^{2} u^{(b)}}{\partial x^{2}}$ and $\frac{\partial^{2} u^{(b)}}{\partial t^{2}}$ coincide.

Setting equal solutions $u_{1}^{(b)}(x, t), u_{2}^{(b)}(x, t)$ and $u_{3}^{(b)}(x, t), u_{4}^{(b)}(x, t)(3.17)$ as well as their first and second-order derivatives on characteristic $x-a t+a b=0$ we find that for continuity of the solution and the first-order derivatives on characteristic $x-a t+a b=0$ the following conditions

$$
\varphi^{(b)}(0)=g^{(b)}(b), \quad \varphi^{(b) \prime}(0)=0
$$

should be fulfilled. For continuity of the second-order derivatives condition

$$
\psi^{(b) \prime}(0)=-\frac{a}{\pi c^{2}}\left(\psi^{(b)}(0)-g^{(b) \prime}(b)+a \varphi^{(b) \prime}(0)\right)\left(1-\frac{c}{\sqrt{a}} \lim _{y \rightarrow 0} \frac{1}{\sqrt{-y}}\right)
$$

should be fulfilled. By the assumptions of theorem $\psi^{(b)}(x) \in C^{1}([0, l])$ that is $\psi^{(b) \prime}(x) \in C([0, l])$ then $\psi^{(b)^{\prime}}(x)$ is limited on interval $[0, l]$ i.e. there exists $C \geqslant 0, C<\infty$ such that $\left|\psi^{(b) \prime}(x)\right|<C$ for all $x \in[0, l]$. But we have that

$$
\left(1-\frac{c}{\sqrt{a}} \lim _{y \rightarrow 0} \frac{1}{\sqrt{-y}}\right) \underset{y \rightarrow 0}{\longrightarrow} \infty .
$$

To solve this contradiction we require that

$$
\psi^{(b)}(0)-g^{(b) \prime}(b)+a \varphi^{(b) \prime}(0)=0 .
$$


Taking into account condition (3.44) and (3.45) we obtain

$$
\psi^{(b)}(0)=g^{(b) \prime}(b), \quad \psi^{(b) \prime}(0)=0 .
$$

Thus for continuity of second-order derivatives conditions (3.46) should be fulfilled.

Setting equal solutions $u_{1}^{(b)}(x, t), u_{3}^{(b)}(x, t)$ and $u_{2}^{(b)}(x, t), u_{4}^{(b)}(x, t)(3.17)$ as well as their first and second-order derivatives on characteristic $l-x-a t+a b=$ 0 we obtain that for continuity of solution, its first-order and second-order derivatives on characteristic $l-x-a t+a b=0$ the following conditions

$$
\varphi^{(b)}(l)=\chi^{(b)}(b), \quad \psi^{(b)}(l)=\chi^{(b) \prime}(b), \quad a^{2} \varphi^{(b) \prime \prime}(l)=\chi^{(b) \prime \prime}(b)
$$

should be fulfilled. Hence for solution continuity up to second-order derivatives conditions (3.44), (3.46) and (3.47) should be fulfilled i.e. coincidence conditions (3.7), (3.8) should be fulfilled.

Theorem 2. If $\varphi^{(b)}(x) \in C^{2}([0, l]), \psi^{(b)}(x) \in C^{1}([0, l]), \chi^{(b)}(t) \in C^{2}\left(\left[b, b+\frac{l}{a}\right]\right)$, $g^{(b)}(t) \in C^{2}\left(\left[b, b+\frac{l}{a}\right]\right)$ and coincidence conditions (3.7), (3.8) are fulfilled then there exists a unique solution $u^{(b)}(x, t) \in C^{2}\left(\bar{\Omega}_{b}\right)$ of initial-boundary value problem (3.1)-(3.4) in domain $\Omega_{b}$ that takes the form (3.17).

Proof. Conditions of theorem yield the conditions of Lemma 1 and Lemma 2. Thus solution $u^{(b)}(x, t)$ of initial-boundary value problem (3.1)-(3.4) exists, takes the form (3.17) and $u^{(b)}(x, t) \in C^{2}\left(\bar{\Omega}_{b}\right)$. Uniqueness of the solution comes from the way of its construction as a particular solution of initial-boundary value problem from general solution of the differential equation.

\section{Solution of Initial-Boundary Value Problem for Wave Equation with Integral Condition by Method of Char- acteristic}

In order to find solution of initial-boundary value problem (2.6)-(2.8) in domain $\Omega$ we divide time frame into intervals with the length $l / a$ (Fig. 3).

Solutions in rectangles $\Omega_{[k]}=\left\{\frac{k l}{a}<t<\frac{(k+1) l}{a}, 0<x<l\right\}, k \in \mathrm{N} \cup$ $\{0\}$ can be found in sequence by formulas (3.17). Here initial and boundary conditions in domain $\Omega_{[0]}$ have the form (2.7), (2.8) and initial and boundary conditions in domain $\Omega_{[k]}, k \in \mathrm{N}$ are found from solution in domain $\Omega_{[k-1]}$ by formulas

$$
\begin{gathered}
u^{[k]}(x, k l / a)=\varphi^{[k]}(x) \equiv u_{4}^{[k-1]}(x, k l / a), \quad 0 \leqslant x \leqslant l, \\
\frac{\partial u^{[k]}(x, k l / a)}{\partial t}=\psi^{[k]}(x) \equiv \frac{\partial u_{4}^{[k-1]}(x, k l / a)}{\partial t}, \quad 0 \leqslant x \leqslant l, \\
u^{[k]}(l, t)=\chi(t), \quad \frac{k l}{a} \leqslant t \leqslant \frac{(k+1) l}{a},
\end{gathered}
$$




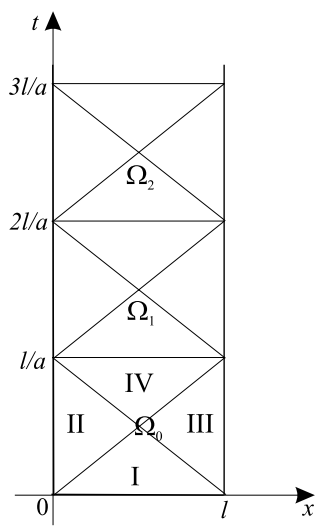

Figure 3. Division of domain $\Omega$ into intervals with the length $l / a$.

$$
u^{[k]}(0, t)=c \int_{\frac{k l}{a}}^{t} \frac{\partial u_{2}^{[k]}(0, \tau)}{\partial x} \frac{d \tau}{\sqrt{t-\tau}}+g^{[k]}(t), \quad \frac{k l}{a} \leqslant t \leqslant \frac{(k+1) l}{a},
$$

where $f^{[k]}=f^{\left(\frac{k l}{a}\right)}$,

$$
g^{[k]}(t)=g(t)+c \sum_{j=0}^{k-1} \int_{j l / a}^{(j+1) l / a} \frac{\partial u_{2}^{[j]}(0, \tau)}{\partial x} \frac{d \tau}{\sqrt{t-\tau}} .
$$

Finally, we have the following theorem.

Theorem 3. If $\varphi(x) \in C^{2}([0, l]), \psi(x) \in C^{1}([0, l]), \chi(t) \in C^{2}([0, \infty)), g(t) \in$ $C^{2}([0, \infty))$ coincidence conditions at the point $(x, t)=(0,0)$

$$
\varphi(0)=g(0), \quad \psi(0)=g^{\prime}(0), \quad \varphi^{\prime}(0)=0, \quad \psi^{\prime}(0)=0
$$

and at the point $(x, t)=(l, 0)$

$$
\varphi(l)=\chi(0), \quad \psi(l)=\chi^{\prime}(0), \quad \chi^{\prime \prime}(0)=a^{2} \varphi^{\prime \prime}(l)
$$

are fulfilled then there exists unique solution $u(x, t) \in C(\Omega)$ of initial-boundary value problem (2.6)-(2.8) in domain $\Omega$. At that in domain $\Omega_{[0]}$ solution $u(x, t) \in C^{2}\left(\bar{\Omega}_{[0]}\right)$ and in domain $\Omega_{[k]}, k \in \mathrm{N}$ solution $u(x, t) \in C^{2}\left(D_{i}^{[k]}\right)$, $i=\overline{1,4}$ and has derivative discontinuity on characteristics $x-$ at $-k l=0$, $(k+1) l-x-a t=0$.

Proof. Conditions of Theorem 2 are fulfilled in domain $\Omega_{[0]}$ thus there exists a unique solution of initial-boundary value problem $(2.6)-(2.8) u(x, t) \in$ $C^{2}\left(\bar{\Omega}_{[0]}\right)$ in domain $\Omega_{[0]}$ and has the form (3.17) when $b=0$.

First, let us show that coincidence conditions (4.5), (4.6) are sufficient for fulfillment of coincidence conditions:

$$
\varphi^{[k]}(0)=g^{[k]}\left(\frac{k l}{a}\right), \quad \varphi^{[k]}(l)=\chi\left(\frac{k l}{a}\right)
$$


in each domain $\Omega_{[k]}, k \in \mathrm{N}$.

We shall use mathematical induction method to prove theorem. In domain $\Omega_{[0]}$ coincidence conditions (4.7) are fulfilled by the theorem data as they correspond to coincidence conditions (4.5), (4.6). We suppose that coincidence conditions (4.7) are fulfilled in domain $\Omega_{[k-1]}$. Let us show that in that case they are also fulfilled in domain $\Omega_{[k]}$.

Expression (4.4) for boundary function $g^{[k]}(t)$ can be presented in the form

$$
g^{[k]}(t)=g^{[k-1]}(t)+c \int_{(k-1) l / a}^{k l / a} \frac{\partial u_{2}^{[k-1]}(0, \tau)}{\partial x} \frac{d \tau}{\sqrt{t-\tau}} .
$$

Let us derive the form of initial and boundary functions $\varphi^{[k]}(x), \psi^{[k]}(x)$, $g^{[k]}(t)$ via functions $\varphi^{[k-1]}(x), \psi^{[k-1]}(x), g^{[k-1]}(t)$. We shall use formulas (4.1), (4.2), (4.8) and solution of initial-boundary value problem (2.6)-(2.8) in domain $\Omega_{[k-1]}$.

$$
\begin{aligned}
& \varphi^{[k]}(x)=\chi\left(\frac{x+(k-1) l}{a}\right)-\varphi^{[k-1]}(l-x)+F^{[k-1]}(x-l), \\
& \psi^{[k]}(x)=\chi^{\prime}\left(\frac{x+(k-1) l}{a}\right)+a \varphi^{[k-1]^{\prime}}(l-x) \\
& -\frac{a^{2}}{\pi c^{2}} g^{[k-1]}\left(\frac{k l-x}{a}\right)+\frac{a^{2}}{\pi c^{2}} F^{[k-1]}(x-l)+\frac{a \sqrt{a}}{\pi c} \\
& \times \int_{0}^{l-x}\left\{\varphi^{[k-1] \prime}(-\xi)+\frac{\psi^{[k-1]}(-\xi)}{a}+\frac{1}{a} g^{[k-1] \prime}\left(b-\frac{\xi}{a}\right)\right\} \frac{d \xi}{\sqrt{\xi-l+x}} \\
& g^{[k]}(t)=g^{[k-1]}(t)+\frac{a}{\pi c} \int_{(k-1) l / a}^{k l / a} g^{[k-1]}(\tau) \frac{d \tau}{\sqrt{t-\tau}}-\frac{2 \sqrt{a}}{\pi} \\
& \times\left\{\frac{a}{\pi c^{2}} g^{[k-1]}((k-1) l / a)+\frac{1}{a} g^{[k-1] \prime}\left(\frac{(k-1) l}{a}\right)-\varphi^{[k-1] \prime}(0)\right. \\
& \left.-\frac{\psi^{[k-1]}(0)}{a}\right\} \int_{(k-1) l / a}^{k l / a} \frac{\sqrt{a \tau-(k-1) l}}{\sqrt{t-\tau}} d \tau-\frac{a}{\pi c} g^{[k-1]}\left(\frac{(k-1) l}{a}\right) \\
& \times \int_{(k-1) l / a}^{k l / a} e^{-\frac{a}{\pi c^{2}}((k-1) l-a \tau)} \operatorname{erfc}\left(\frac{\sqrt{a(a \tau-(k-1) l)}}{\sqrt{\pi} c}\right) \frac{d \tau}{\sqrt{t-\tau}} \\
& -\frac{2 \sqrt{a}}{\pi} \int_{0}^{-l}\left\{-\frac{1}{\pi c^{2}} g^{[k-1] \prime}\left(\frac{(k-1) l-\xi}{a}\right)-\frac{1}{a^{2}} g^{[k-1] \prime \prime}\left(\frac{(k-1) l-\xi}{a}\right)\right. \\
& \left.+\varphi^{[k-1] \prime \prime}(-\xi)+\frac{\psi^{[k-1] \prime}(-\xi)}{a}\right\} \int_{(k-1) l-\xi / a}^{k l / a} \frac{\sqrt{\xi+a \tau-(k-1) l}}{\sqrt{t-\tau}} d \tau d \xi \\
& -\frac{a}{\pi c} \int_{0}^{-l}\left\{\frac{a}{\pi c^{2}} g^{[k-1]}\left(\frac{(k-1) l-\xi}{a}\right)-\varphi^{[k-1] \prime}(-\xi)-\frac{\psi^{[k-1]}(-\xi)}{a}\right\} \\
& \times \int_{(k-1) l-\xi / a}^{k l / a} e^{-\frac{a}{\pi c^{2}}((k-1) l-a \tau-\xi)} \operatorname{erfc}\left(\frac{\sqrt{a(\xi+a \tau-(k-1) l)}}{\sqrt{\pi} c}\right) \frac{d \tau}{\sqrt{t-\tau}} d \xi
\end{aligned}
$$

Substituting expressions (4.9), (4.11) into coincidence condition (4.7) and performing transformations, we prove fulfillment of coincidence condition (4.7) 
in domain $\Omega_{[k]}$. Similarly substituting expression (4.9) into the second condition condition (4.7) we prove fulfillment of coincidence conditions (4.7) in domain $\Omega_{[k]}$ under the assumption that coincidence conditions (4.7) are fulfilled in domain $\Omega_{[k-1]}$. Thus conditions of Lemma 1 are fulfilled in domain $\Omega_{[k]}$, $k \in \mathrm{N}$. Hence there exists unique solution $u(x, t) \in C\left(\bar{\Omega}_{[k]}\right), u(x, t) \in C^{2}\left(D_{i}^{[k]}\right)$, $i=\overline{1,4}$ in domain $\Omega_{[k]}$ and has the form (3.17) when $b=k l / a$.

We can show that coincidence conditions $\psi^{[k]}(0)=g^{[k] \prime}\left(\frac{k l}{a}\right), \varphi^{[k] \prime}(0)=0$, $\psi^{[k] \prime}(0)=0$ are not fulfilled in the general case in domain $\Omega_{[k]}$ when they are fulfilled in domain $\Omega_{[k-1]}, k \in \mathrm{N}$. Then according to proof of Lemma 3 solution $u(x, t) \notin C^{1}\left(\Omega_{[k]}\right)$.

Thus solution $u(x, t) \in C\left(\bar{\Omega}_{[k]}\right), u(x, t) \in C^{2}\left(D_{i}^{[k]}\right), i=\overline{1,4}$ and first-order and second-order derivatives has discontinuity on characteristics $x-a t+k l=0$, $l-x+a t+k l=0, k \in \mathrm{N}$. This solution is obviously unique in each domain $D_{i}^{[k]}$.

Let us show that derivatives up to second-order remain continuous when coming through border $t=k l / a$ between domains $\Omega_{[k-1]}$ and $\Omega_{[k]}$. Continuity of solution and first-order time derivative obviously comes from expression for initial functions $\varphi^{[k]}(x) \quad \psi^{[k]}(x)(4.1),(4.2)$.

Let us prove a continuity of the first-order derivative by $x$.

$$
\begin{aligned}
\frac{\partial u_{1}^{[k]}(x, k l / a)}{\partial x}= & \left\{\frac{\varphi^{[k] \prime}(x-a t+k l)+\varphi^{[k] \prime}(x+a t-k l)}{2}\right. \\
& \left.+\frac{\psi^{[k]}(x+a t-k l)-\psi^{[k]}(x-a t+k l)}{2 a}\right\}\left.\right|_{t=k l / a}=\varphi^{[k] \prime}(x) .
\end{aligned}
$$

Using identity (4.1) we obtain

$$
\frac{\partial u_{1}^{[k]}(x, k l / a)}{\partial x}=\varphi^{[k] \prime}(x)=\frac{\partial u_{4}^{[k-1]}(x, k l / a)}{\partial x},
$$

that means that the first-order derivative by $x$ is continuous. Let us prove a continuity of the second-order derivative by $x$.

$$
\begin{aligned}
\frac{\partial^{2} u_{1}^{[k]}(x, k l / a)}{\partial x^{2}}= & \left\{\frac{\varphi^{[k] \prime \prime}(x-a t+k l)+\varphi^{[k] \prime \prime}(x+a t-k l)}{2}\right. \\
& \left.+\frac{\psi^{[k] \prime}(x+a t-a b)-\psi^{[k] \prime}(x-a t+k l)}{2 a}\right\}\left.\right|_{t=k l / a}=\varphi^{[k] \prime \prime}(x) .
\end{aligned}
$$

Using identity (4.1) we obtain

$$
\frac{\partial^{2} u_{1}^{[k]}(x, k l / a)}{\partial x^{2}}=\varphi^{[k] \prime \prime}(x)=\frac{\partial^{2} u_{4}^{[k-1]}(x, k l / a)}{\partial^{2} x},
$$

that means that second-order derivative by $x$ is continuous. Let us show a 
continuity of the second-order derivative by $t$.

$$
\begin{aligned}
\frac{\partial^{2} u_{1}^{[k]}(x, k l / a)}{\partial t^{2}}= & \left\{a^{2} \frac{\varphi^{[k] \prime \prime}(x+a t-k l)+\varphi^{[k] \prime \prime}(x-a t+k l)}{2}\right. \\
& \left.+a \frac{\psi^{[k] \prime}(x+a t-k l)-\psi^{[k] \prime}(x-a t+k l)}{2}\right\}\left.\right|_{t=k l / a}=a^{2} \varphi^{[k] \prime \prime}(x) .
\end{aligned}
$$

Using identity (4.1) we get

$$
\frac{\partial^{2} u_{1}^{[k]}(x, k l / a)}{\partial t^{2}}=a^{2} \varphi^{[k] \prime \prime}(x)=a^{2} \frac{\partial^{2} u_{4}^{[k-1]}(x, k l / a)}{\partial x^{2}} .
$$

Using identity (2.6) we obtain

$$
\frac{\partial^{2} u_{1}^{[k]}(x, k l / a)}{\partial t^{2}}=a^{2} \frac{\partial^{2} u_{4}^{[k-1]}(x, k l / a)}{\partial x^{2}}=\frac{\partial^{2} u_{4}^{[k-1]}(x, k l / a)}{\partial t^{2}},
$$

that means that second-order derivative by $t$ is continuous.

Let us prove a continuity of the mixed derivative.

$$
\begin{aligned}
\frac{\partial^{2} u_{1}^{[k]}(x, k l / a)}{\partial x \partial t}= & \left\{a \frac{\varphi^{[k] \prime \prime}(x+a t-k l)-\varphi^{[k] \prime \prime}(x-a t+k l)}{2}\right. \\
& \left.+\frac{\psi^{[k] \prime}(x+a t-k l)+\psi^{[k] \prime}(x-a t+k l)}{2}\right\}\left.\right|_{t=k l / a}=\psi^{[k] \prime}(x) .
\end{aligned}
$$

Using identity (4.2) we get

$$
\frac{\partial^{2} u_{1}^{[k]}(x, k l / a)}{\partial x \partial t}=\psi^{[k] \prime}(x)=\frac{\partial^{2} u_{4}^{[k-1]}(x, k l / a)}{\partial x \partial t},
$$

that means that mixed derivative is continuous. Hence solution is continuous up to the second-order derivatives when coming through borders of domain.

Thus we have proved that solution $u(x, t) \in C^{2}\left(\bar{\Omega}_{[0]}\right), u(x, t) \in C\left(\bar{\Omega}_{[k]}\right)$, $k \in \mathrm{N}, u(x, t) \in C^{2}\left(D_{i}^{[k]}\right), i=\overline{1,4}$ and first-order and second-order derivatives have discontinuity on characteristics $x-a t+k l=0, l-x+a t+k l=0$.

Hence we have proved the unique existence of classical solution of initialboundary value problem (2.6)-(2.8) when coincidence conditions (4.5), (4.6) were fulfilled. We have also found analytical solution of the problem.

\section{References}

[1] M. Abramovits and I. Stigan. Reference-Book for Special Functions. Science, Moscow, 1979. (in Russian)

[2] S.M. Apollonskiy and V.T. Erofeenko. Equivalent Boundary Conditions in Electrodynamics. Bezopasnost, St. Petersburg, 1999. (in Russian)

[3] V.B. Dmitriev. Nonlocal problem with integral condition for hyperbolic equation. Bulletin of Samara Technical University, 42:35-40, 2006. (in Russian) 
[4] V.B. Dmitriev. Nonlocal problem with integral conditions for wave equation. Bulletin of Samara State University, 2(42):15-27, 2006. (in Russian)

[5] E.A. Eremin and M.V. Urev. Generalized statement of initial-boundary value problem for Maxwell's system with new boundary conditions. Proceedings of Russian Academy of Science. Comp. Math., 5:109-115, 1996. (in Russian)

[6] V.T. Erofeenko, V.I. Korzyuk and J.V. Pulko. Characteristics method for initial boundary-value problem for wave equation with boundary condition. First International Conference Mathematical Modeling and Differential Equations, p. 53, 2008.

[7] V.T. Erofeenko and I.S. Kozlovskaya. Mathematical Models in Electrodynamics. Part 2. Belarusian State University Publisher, Minsk, 2008. (in Russian)

[8] D.G. Gordeziani and G.A. Avalishvili. Solution of nonlocal problem for onedimensional string oscillation. Math. Modeling, 12(1):94-103, 2000. (in Russian)

[9] V.I. Korzyuk, V.T. Erofeenko and J.V. Pulko. Classical solution for wave equation for initial-boundary value problem with integral with respect to time boundary condition. Reports of Belarusian National Academy of Science, 53(5):36-41, 2009. (in Russian)

[10] V.I. Korzyuk, V.T. Erofeenko and J.V. Pulko. Initial-boundary value problem for wave equation with fractional derivative in boundary condition. AMADE2009, pp. 60-61, 2009. (in Russian)

[11] V.I. Korzyuk and J.V. Pulko. Initial-boundary value problem for wave equation with integral boundary condition. Abstracts of 13th International Conference 'Mathematical Modelling and Analysis' (Jule 4-7, 2008, Tartu (Kaariku), Estonia), pp. 60-61, 2009. (in Russian)

[12] A.I. Kozhanov and L.S. Pulkina. Boundary-value problems with integral condition for multidimensional hyperbolic equations. Russian Academy of Science Reports, 404(5):589-592, 2005. (in Russian)

[13] V.F. Kravchenko and V.T. Erofeenko. About impedance boundary conditions taking into account curvature of surface. Pyrotechnics and Electronics, 45(11):1300-1306, 2000. (in Russian)

[14] L.S. Pulkina. About solubility of nonlocal problem with integral boundary conditions for hyperbolical equation. Bulletin of Samara State University, 2(80):63-67, 1998. (in Russian)

[15] L.S. Pulkina. Mixed problem with integral condition for hyperbolic equation. Math. Notes, 74(3):435-445, 2003. (in Russian)

[16] L.S. Pulkina. Nonlocal problem with integral conditions for hyperbolic equation. Differ. Equ., 40(7):887-892, 2004. (in Russian)

[17] L.S. Pulkina. Nonlocal problem with integral condition of the first kind for multidimensional hyperbolic equation. Russian Academy of Science Reports, 416(5):597-599, 2007. (in Russian)

[18] M.V. Urev. Boundary conditions for Maxwell's equations with arbitrary time dependance. J. Comput. Math. and Math. Phys., 37(12):1489-1497, 1997. (in Russian) 